\title{
EVALUACIÓN DE LA DOCENCIA EN ENFERMERÍA DESDE LA OPINIÓN DE LOS ACTORES DE LA EDUCACIÓN
}

Mtra. Gandhy Ponce Gómez*, Mtra. Liliana Gonzólez Juárez** y Mtra. Martha Lilia Bernal Becerri|***

* Profesora de Asignatura "B " de lo Escuela Nacional de Enfermeria y Obstetricia, ** Profesora de Carrera Titular "A" de la Escuela Nacional de Enfermeria y Obstetricia., "." Profesora de Asignatura "B" de la Escuela Nacional de Enfermeria y Obstetricia.

\section{RESUMEN.}

La práctica docente como praxis social, debe trascender de dimensión para reconocer la relación entre personas, mediar un saber colectivo culturalmente organizado e intrínsecamente conectado con un conjunto de valores personales y sociales implicando su transformación.

En Enfermeria la evaluación docente cobra relevancia, no sólo por cuantificar un actuar en lo didáctico, sino de indagar la dimensión personal, interpersonal, social y de valores que se forma en estudiantes; porque se trata de transformadores sociales que serán responsables del cuidado a la salud de los individuos.

Metodologia: estudio de tipo cuantitativo correlacional, en alumnos y docentes de una escuela de Enfermerla de nivel superior en el DF (" $n$ " de docentes $=23$ y " $n$ " de alumnos $=250$ ).

Resultados: Los profesores de $2^{\circ}$ y $8^{\circ}$ semestre son evaluados más alto con respecto al índice general de desempeño; la dimensión didáctica es la evaluada con medias más bajas en general, y que aún con una relación relativamente baja son los docentes de menor edad quienes reciben evaluaciones más favorables.

Transitando desde la autovaloración y la perspectiva del alumno hacia el descubrimiento de la riqueza y complejidad de la docencia se pretende transformar reflexiva y críticamente la importante tarea educativa.

PALABRAS CLAVE: Desempeño Docente, Evaluación de la Docencia "Docencia, Alumno, Evaluación, Autoevaluación.

\section{ABSTRACT.}

Teaching practice as a social praxis, must transcend dimensions for recognizing the relationship between people, for mediating a collective knowledge culturally organized and intrinsically connected with a personal and social values array that implies its transformation.

In nursing, teaching evaluation takes relevance, not only because it quantifies an action within a didactical scope, but for inquiring personal interpersonal, social and values dimentions that are formed in students; becouse it is about social transformers who would be responsible for individuals' health care.

Methodology. Correlated quantitative study with students and teachers in a high level nursing school within D.F. area (" $n$ " of teachers $=23$; " $n$ " of students $=250$ ).

Outcomes. 2nd. and 8th. Semester teachers are evaluated higher regarding the performance general index; didactical dimension is in general evaluated with lower measures, and, even with a relatively lower relation, are the less age or younger teachers who receive more favorable evaluations.

Moving from students' self-evaluation and perspective toward the discovery of the richness and complexity of teaching, it is pretended to transform in a reflexive and critically way, the important educational tosk.

Key words. Teaching performance, teaching evaluation, teaching, student, evaluation, self-evaluation. 


\section{INTRODUCCION}

A la docencia en Enfermería le implica un fuerte reto, al enfrentarse a la crisis social existente, con grandes tensiones económicas, culturales y políticas que ponen a prueba su fortaleza y de la educación en general. La responsabilidad del docente es de preparar el recurso humano que sea capaz de ejecutar eficiente y competentemente su profesión, para cumplir el rol de agente transformador social en el ámbito de cuidado a la salud de los individuos, que se diferencie de "una Enfermería sin conciencia, donde se aprenden técnicas instrumentales deshistorizadas y desarticuladas de una visión comprensiva del quehacer"), de una Enfermería aislada del cuidado.

El modelo formativo e integral que se aplicó es una autoevaluación del docente y evaluación por parte del alumnado, con el objeto de establecer posibles relaciones entre dimensiones didáctica, personal, interpersonal, social y de enseñanza de valores, con variables sociodemográficas de ambas poblaciones, con las cuales los docentes puedan identificar los aspectos que podrían ser perfeccionados en su práctica.

Se coincide con Fierro, Fortoul y Rosas², al ubicar la labor docente en función de cinco dimensiones: personal, interpersonal, social, didáctica y de enseñanza de valores. Se hace notar que ellos incluyen además la esfera institucional, pero que no será estudiada en este momento para la problemática en estudio. Así tenemos:

Dimensión personal: Se reconoce al docente como un ser histórico capaz de analizar su presente y construir su futuro, recuperar la forma en que se enlazan su historia personal y su trayectoria profesional, su vida cotidiana y trabajo en la institución educativa.

Dimensión interpersonal: Son las relaciones siempre complejas, que se construyen sobre la base de las diferencias individuales en un marco institucional; estas diferencias no solamente atañen a la edad, el sexo o la escolaridad, sino a cuestiones menos evidentes a primera vista pero de igual o mayor importancia: la diversidad de metas, los intereses, las ideologías frente a la enseñanza y las preferencias políticas, por ejemplo.

Dimensión social: El quehacer docente se desarrolla en un entorno histórico, político, social, geográfico, cultural y económico particular, que le imprime ciertas exigencias y que al mismo tiempo es el espacio de incidencia más inmediato de su labor; pero además se debe resaltar el alcance social que tiene la práctica educativa, ya que los productos en los estudiantes serán expresados en los profesionales que emerjan del proyecto educativo al que pertenezcan.

Dimensión Didáctica: Son las habilidades para comunicar un tema, hacer inteligible un asunto difícil y lograr un aprendizaje en los alumnos. Implica tanto las habilidades del docente, como el uso correcto de estrategias de enseñanza que guien al estudiante en el proceso enseñanza aprendizaje. El docente debe proveer de herramientas al estudiante para la construcción del conocimiento, tales como la reflexión, el análisis, las apropiaciones significativas, saberes integrados y aterrizados en el plano de lo real, creatividad, innovación, así como de secuencias previas que lo lleven al razonamiento lógico.

Dimensión de enseñanza de valores: En México, a consecuencia de la mayor escolaridad de la población, las crecientes manifestaciones culturales, la apertura a otras sociedades y culturas, la mayor fuerza de su población joven; etc., han venido ganando espacio los valores de la libertad, responsabilidad, solidaridad, justicia social, respeto y tolerancia a las diferencias, búsqueda de la verdad, respeto a los derechos humanos, conservación del medio ambiente y una cultura de paz, que el docente debe verter en forma conciente a sus estudiantes.

De la manera en que cada profesor logre armonizar e integrar estas dimensiones promoverá una relación liberadora en la que se recree el conocimiento sobre la base del respeto y el apoyo mutuos en el proceso de desarrollo personal.

\section{MATERIAL Y MÉTODOS}

Se trata de un estudio cuantitativo y transversal correlacional, ya que pretende evaluar el desempeño del docente en Enfermería a partir de la opinión de los actores del proceso (alumnos y docentes), desde las dimensiones didáctica, personal, interpersonal, social y de enseñanza de valores.

La población de estudio se constituyó de alumnos del sistema escolarizado de una Escuela de Enfermeria de nivel superior, de los semestres $2^{\circ}, 4^{\circ}, 6^{\circ}$, y $8^{\circ}$. Este reporte hace un análisis de la opinión del 86\% $(n=530)$ de los alumnos de la población original inscrita en el turno matutino $(N=616)$, y de 23 docentes de enfermería, quienes comprenden el $96 \%$ de la población original de docentes de Enfermería del mismo turno $(\mathrm{N}=24)$.

Se hizo uso de la escala tipo Likert, con valores de 1 a 5 , donde 1 es nunca y 5 siempre lo realiza. Su medición fué 
en un índice de 1 a 5, donde 1 es el nivel más bajo y significa desempeño inadecuado y 5 es el nivel más alto, que quiere decir un desempeño adecuado (Cuadro 1$)^{3}$.

Se incluyeron variables intervinientes para la población docente tales como antigüedad, categoría que ocupa, edad, escolaridad y semestre en que imparte cátedra, etc; mientras que las variables intervinientes para la población de estudiantes implican sexo, edad, escuela de bachillerato de procedencia, semestre, promedio de semestres anteriores y calificación que esperaba obtener al final de la asignatura.

El instrumento fué adaptado por Ponce del original de Espino, Curtidor y Uvalle 4 para "Medir la Calidad del Desempeño Docente de Enfermería en el Aula". Se llevó a cabo un jueceo de expertos en la materia y una prueba piloto en poblaciones de docentes y alumnos con características similares a las del estudio. En la fase de ejecución después de aplicado el instrumento a la población de 530 estudiantes, la prueba de confiabilidad alcanzó un Alpha de Cronbach de .9647; mientras que el de docentes (23 individuos) obtuvo .8833 .

Los datos fueron procesados en el paquete estadistico SPSS V.1 1.0. Se realizó un análisis descriptivo y un análisis inferencial con problemas de comparación y relación por medio de las pruebas ANOVA y de " $r$ " de Pearson.

\section{RESULTADOS}

Se observó en la evaluación de los docentes en Enfermería por parte de la opinión de los alumnos por dimensiones, que las medias más altas se encuentran en las dimensiones personal y valoral (3.59 y 3.58 respectivamente), lo que muestra que los alumnos tienen una percepción de sus docentes con "desempeño regular" y los evalúan ligeramente más bajos en la dimensión didáctica (3.21, es decir desempeño regular). En cuanto al indice general los alumnos los evalúan alcanzando un indice de 3.47 que también los posiciona como docentes con un "desempeño regular".

Respecto a la autoevaluación de los docentes en Enfermería por dimensiones, las medias más altas se encuentran en las dimensiones social y valoral (4.44 y 4.37 respectivamente). Lo que muestra que los docentes se perciben a sí mismos con un desempeño adecuado en dimensiones en las que interactúan con sus alumnos, y se califican ligeramente más bajos en la dimensión didáctica (3.92, es decir desempeño regular). En cuanto al indice general los docentes se autoevalúan alcanzando un índice de 4.29, que también los cataloga como docentes con un desempeño adecuado (Cuadro No. 2).

De acuerdo con el análisis inferencial en lo que respecta a la diferencia de la evaluación general que otorga el alumno al docente (Media $=3.47$, desempeño regular) se tiene que ésta, no es igual en los distintos semestres (prob. $.000<0.05[\mathrm{~F}=36.206 \mathrm{gl}$ intergupos $=3, \mathrm{gl}$ intragupos $=$ 526]). Se encontró que el desempeño de los docentes de $8^{\circ}$ es más alto (Media $=4.12$ ) y el índice más bajo es el que se le da a los docentes de $4^{\circ}$ semestre (Media $=$ 3.15).

En cuanto al Índice de desempeño asignado al docente Enfermero por parte del alumno por dimensiones, se tiene que las áreas personal y valoral, son las que obtienen las medias más altas, en el $8^{\circ}$ semestre. Y la media más baja en que son evaluados es en la dimensión interpersonal. Mientras que en los semestres de $4^{\circ}$ y $6^{\circ}$. Observaron las medias más bajas en las cinco dimensiones. (ver Cuadro No. 3)

En cuanto a la comparación de "nivel de escolaridad" con "índice general de desempeño docente desde la opinión del alumno" se hallaron diferencias estadísticas por medio de ANOVA $(F=3.4567, p<.05)$ que muestran que los docentes con licenciatura son mejor evaluados en las dimensiones valoral, personal e interpersonal (Medias $=3.38,4.21$ y 4.34 respectivamente); mientras que los profesores con Maestría son evaluados más altos en la dimensión didáctica (Media $=4.02$ ).

En la relación de edad del docente con la evaluación que el alumno otorga existe un relación inversamente proporcional baja ( $r p=-.467$, prob. $=.025)$. Lo que muestra que sólo en el caso de algunos docentes a menor edad, los alumnos le otorgan mayor calificación.

\section{DISCUSIÓN}

La media de los docentes en su desempeño general los ubica en un nivel de desempeño regular, lo que significa que su actividad no tiende a ser constante, que por su inconsistencia cabe la posibilidad de llegar a pasar desapercibida o intrascendente para los alumnos. Cashin ${ }^{5}$, explica parte de estas diferencias por medio de la orientación cuantitativa versus cualitativa: entre más cuantitativo sea el curso o disciplina, más severa será la evaluación del profesor por los estudiantes, de ahí que los maestros de las ciencias o ingenierías sean evaluados con menores promedios que las otras disciplinas. Así, los docentes de Enfermería en que se admite cierta comple- 
jidad, lo mismo que contenidos de carácter humanístico, es concebido que sean evaluados en rangos medios.

Respecto a la baja relación entre edad y eficiencia docente, apoyan los resultados encontrados por Feldman ${ }^{6}$, que indican que los docentes jóvenes son mejor evaluados en aspectos tales como entusiasmo por la materia, claridad, entendimiento y ayuda. En el caso de los profesores más experimentados, se da una relación inversa con las dimensiones de claridad, entusiasmo, apertura hacia las opiniones de otros, de la rnisma forma que en el estudio de Renaud y Murray ${ }^{7}$ donde los rasgos de relación interpersonal varían de acuerdo a la edad del profesor y algunos de ellos declinan con la adultez.

En relación al nivel de escolaridad de los docentes, Cruz, Crispín y Ávila ${ }^{8}$ encontraron que los profesores de licen. ciatura dominan la materia, pero necesitan más apoyo en lo que se refiere a la metodología de enseñanza-aprendizaje y su fortaleza se encuentra en la promoción y atención a las expresiones de los estudiantes. Por su parte, los profesores con posgrado tienen como fortaleza el dominio de la materia y debilidad en las relaciones interpersonales. Esta revisión podría apoyar los hallazgos en cuanto a que son los docentes con licenciatura quienes son evaluados más alto en las dimensiones de tipo social, mientras que los docentes con posgrado son evaluados mejor en lo didáctico.

\section{CONCLUSIONES}

La enseñanza del cuidado, no es una pedagogía más; sino que atiende a una complejidad cultural e histórica, debido a que la Enfermería se convirtió en una práctica social identificada con el humanismo. Todo docente debería tener presente que educar implica no sólo socializar el conocimiento, sino introducirse en el significado de la cultura en la cual se está inmerso, contextualizarse en las estructuras propias de docentes y alumnos.

La Enfermería, como disciplina profesional involucra dos componentes: como disciplina se centra en el cuidado de la salud de los seres humanos en forma integral y en relación con su ambiente; y como profesión hace esfuerzos para mejorar la calidad de vida de los individuos a través de tratar de resolver los problemas o necesidades que interfieren en esa calidad. Para desarrollar esos dos componentes, González ${ }^{9}$ asegura que es indispensable generar conocimientos, transmitirlos y transformarlos, mediante una práctica docente participativa, activa, integral y totalizadora, es decir ética, en virtud de que formamos seres humanos para cuidar seres humanos.

\section{REFERENCIAS BIBLIOGRÁFICAS}

1. Castrillón - C. S. "Lo educación y el cuidado de enfermeria". En: Grupo de Cuidado. Dimensiones del cuidado. Facultad de Enfermería. Universidad Nacional de Colombia. Bogotá, 1997. p. 35.

2. Fierro - C., Fortoul - B., \& Rosas - L. Transformando la Práctica Docente: Una propuesta basada en la investigación - acción. Maestro y Enseñanza. México, 2002. p. 28 - 37.

3. Loredo - E J. \& Grijalva - M, O. Propuesto de un instrumento de evaluación de la docencia para los estudios de posgrado. En: Rueda, B. \& Díaz B. Evaluación de la Docencia. Perspectivas actuales. México, 2002. p. $108-110$.

4. Espíno - V. E., Curtidor - S. B. \& Uvalle, VM. Instrumento para Medir la Calidad del Desempeño Docente de Enfermería en el Aula. En la Revista Enfermeras, Número XXXV. México, 2000. p. 54 - 59.

5. Cashin - W. E. "Students do rate different academia fields differently". [en: García -C. J. ¿Qué factores extraclase o sesgos afectan la evaluación docente en la educación superior?. En la Revista Mexicana de Investigación Educativa. Vol. 5 (10). Disponible en World Wide WEB: http://muw.comie.org.mx/revista/Resumenes/Numero 10/10res2.htm

6. Feldman - K. A. "Course characteristics and college students? Ratings of theirs teachers and courses: What we know and what we don't. En: García, GJ. ¿Qué factores extraclase o sesgos afectan la evaluación docente en la educación superior?. En la Revista Mexicana de Investigación Educativa. Vol. 5 (10). Disponible en la World Wide Web. http://unw.comie.org.mx/revista/Resumenes/Numero 10/10res2.htm

7. Renaud - R. D y Murray - H. A. Aging, personality, and teaching effectiveness in academia psychologists". En: García, GJ. ¿Qué factores extraclase o sesgos afectan la evaluación docente en la educación superior?. En la Revista Mexicana de Investigación Educativa vol. 5 (10). Disponible en la World Wide Web http://unw.comie.org.mx/revista/Resumenes/Numero 10/10res2.htm

8. Cruz - R. I, Crispín - B. M, Avila - R. H. La Evaluación formativa Estrategia para promover el cambio y mejorar la docencia. En: Rueda, BM. \& Díaz Barriga, F. Compiladores. Evaluación de la docencia. Perspectivas actuales. Buenos Aires, 2000

9. González - V. \& Grande - Q. Experiencia en la evaluación de la Universidad: El caso del profesorado. En la Revista Electrónica Interuniversitaria de Formación del Profesorado, 34. Disponible en la World Wide Web: http://umw.revie.interuni.profesorado/34.html

\section{DIRECCIÓN PARA CORRESPONDENCIA}

Mtra. Gandhy Ponce Gómez: gandhy_ponce@yahoo.com.mx

Mtra. Liliana González Juárez:llili|@hotmail.com.

Mtra. Martha Lilia Bernal Becerril: marthaliliabb@hotmail.com

\section{FUENTES DE APOYO:}

Escuela Nacional de Enfermería y Obstetricia.

Programa de Apoyo para la Superación del Personal Académico de la UNAM (PASPA) 


\section{CUADRO No. 1 \\ ÍNDICE DE DESEMPEÑO DOCENTE}

\begin{tabular}{|c|c|c|c|}
\hline $\begin{array}{l}\text { INTERVALOS } \\
\text { EN LA } \\
\text { ESCALA }\end{array}$ & $\begin{array}{c}\text { TIPO } \\
\text { DE } \\
\text { DESEMPEÑO }\end{array}$ & VALORACIÓN & $\begin{array}{l}\text { CRITERIOS } \\
\text { DE } \\
\text { DECISIÓN }\end{array}$ \\
\hline $4.1-5$ & $\begin{array}{l}\text { Desempeño } \\
\text { Adecuado }\end{array}$ & $\begin{array}{l}\text { Este nivel de actividad conlleva la ejecución de competencias } \\
\text { profesionales continuas, justificadas y adaptadas al momento } \\
\text { del proceso educativo que caracterizan a la docencia (activi- } \\
\text { dades de planeación, competencias profesionales, comunica- } \\
\text { ción, estrategias didácticas y el manejo ético o de valores). }\end{array}$ & $\begin{array}{l}\text { Debe estimular estos comporta- } \\
\text { mientos y establecer las condicio- } \\
\text { nes para mantenerlos e incluso } \\
\text { potenciarlos. }\end{array}$ \\
\hline $3.1-4$ & $\begin{array}{l}\text { Desempeño } \\
\text { Regular }\end{array}$ & $\begin{array}{l}\text { Este nivel de ejecución no tiende a ser regular en sus inter- } \\
\text { venciones cotidianas y en las dimensiones descritas. Su acti- } \\
\text { vidad puede llegar a pasar desapercibida o intrascendente } \\
\text { para los alumnos. }\end{array}$ & $\begin{array}{l}\text { Es recomendable analizar con el } \\
\text { docente y los alumnos aquellas } \\
\text { áreas que podrian corregirse. }\end{array}$ \\
\hline $2.1-3$ & $\begin{array}{l}\text { Desempeño } \\
\text { con carenclas }\end{array}$ & $\begin{array}{l}\text { Un desempeño con carencias no refleja la planeación o el } \\
\text { dominio de las competencias propias de un docente en lo } \\
\text { didáctico, social o del aspecto ético; y por lo tanto no pro- } \\
\text { mueve el desarrollo integral de la personalidad tanto del } \\
\text { docente como del alumno. }\end{array}$ & $\begin{array}{l}\text { Análisis de la actuación docente en } \\
\text { relación con los alumnos, experien- } \\
\text { cia y formación docente. }\end{array}$ \\
\hline $1-2$ & $\begin{array}{l}\text { Desempeño } \\
\text { Inadecuado }\end{array}$ & $\begin{array}{l}\text { Este desempeño no corresponde de ninguna forma a los } \\
\text { propósitos de reunión de alumno y docente para el proceso } \\
\text { enseñanza - aprendizaje. Posee serias deficiencias y trae } \\
\text { resultados contraproducentes tanto en el docente como en } \\
\text { el alumno tales como la desatención, la inasistencia y la falta } \\
\text { de motivantes para el desarrollo de ambos actores. }\end{array}$ & $\begin{array}{l}\text { Se requiere un estudio amplio de lo } \\
\text { ocurido en el curso y del perfil del } \\
\text { docente. }\end{array}$ \\
\hline
\end{tabular}

Cuadro No. 1. Loredo - E J. \& Grijalva - M, O. (2000). Propuesta de un instrumento de evaluación de la docencia para los estudios de posgrado.

CUADRO NO. 2

AUTOEVALUACIÓN POR DIMENSIONES

\begin{tabular}{|lll|}
\hline Índice por dimension & Media & D.E. \\
Personal & 4,35 &, 36 \\
\hline Interpersonal & 4,34 &, 36 \\
\hline Didáctica & 3,92 &, 41 \\
\hline Enseñanza de valores & 4,37 &, 41 \\
Social & 4,44 &, 34 \\
\hline Índice Clobal & 4,29 &, 30 \\
\hline
\end{tabular}

CUADRO NO. 3

ÍNDICE DE DESEMPEÑO DOCENTE EVALUADA POR ALUMNOS / SEMESTRE

\begin{tabular}{|c|c|c|c|c|c|c|c|}
\hline & & & emestre ( & ias) & & & \\
\hline DIMENSIÓN & $\begin{array}{c}20 \\
N=179\end{array}$ & $\begin{array}{c}40 . \\
N=149\end{array}$ & $\begin{array}{c}60 . \\
N=133\end{array}$ & $\begin{array}{c}8^{\circ} \\
N=69\end{array}$ & Total & $F$ & Prob. \\
\hline $\begin{array}{l}\text { Índice general } \\
\text { de desempeño }\end{array}$ & 3.67 & 3.15 & 3.24 & 4.12 & 3.47 & 3.20 & .000 \\
\hline Didáctica & 3.42 & 2.82 & 3.02 & 3.87 & 3.21 & 43.74 & .000 \\
\hline Valoral & 3.75 & 3.33 & 3.29 & 4.22 & 3.58 & 24.15 & .000 \\
\hline Personal & 3.75 & 3.30 & 3.39 & 4.22 & 3.59 & 23.97 & .000 \\
\hline Interpersonal & 3.76 & 2.99 & 3.19 & 4.17 & 3.45 & 43.64 & .000 \\
\hline Social & 3.66 & 3.31 & 3.31 & 4.10 & 3.53 & 22.88 & .000 \\
\hline
\end{tabular}

